Original Review Paper

\title{
Effects of Dietary Nucleotides on Immune Mechanisms and Physical State in Children with Chronic Respiratory Problems
}

\author{
${ }^{1}$ Richter Josef, ${ }^{2}$ Svozil Vladimir, ${ }^{1}$ Král Vlastimil, \\ ${ }^{1}$ Rajnohová Dobiášová Lucie, ${ }^{1}$ Stiborová Ivana, ${ }^{1}$ Pohořská Jitka and ${ }^{3}$ Vetvicka Vaclav \\ ${ }^{I}$ Zdravotni Ústav se Sidlem v Usti nad Labem, Czech Republic \\ ${ }^{2}$ Sanatorium Edel, Zlaté Hory, Czech Republic \\ ${ }^{3}$ Department of Pathology, University of Louisville, Louisville, KY, USA
}

\author{
Article history \\ Received: 08-04-2014 \\ Revised: 21-05-2015 \\ Accepted: 21-05-2015 \\ Corresponding Author: \\ Vetvicka Vaclav \\ Department of Pathology, \\ University of Louisville, \\ Louisville, KY, USA \\ Email: vaclav.vetvicka@louisville.edu
}

\begin{abstract}
Heavy industrial pollution and/or passive smoking negatively affect the overall immune health of a population. We used a model of children with chronic respiratory problems and evaluated the effects of short term supplementation of their diet with nucleotides. We measured the level of sIgA, eNO levels and total physical activity. We found positive effects of oral supplementation with commercially available nucleotides. Our data demonstrate strong improvements in physical activity, strong decrease of eNO levels and maintaining of the levels of sIgA. We conclude that supplementation with nucleotides offers an easy way to improve health conditions in children.
\end{abstract}

Keywords: Nucleotides, sIgA, Immunity, Children, Physical Activity, eNO

\section{Introduction}

A long-term focus of our laboratory is the research of how the environmental conditions affect the health of population. We are particularly interested in the possible regeneration of physical status of children exposed to environmental pollution of both internal and external environment (Lee et al., 2013; Richter et al., 2014; Vaclav et al., 2013a; 2013b; 2013c). We are focused most of all on children with chronic respiratory problems caused by living in industrial regions of northern Bohemia and northern Moravia where the extremely high concentration of environmental contamination helped to gain the term Black Spot of Europe. The fact that this region is populated mostly by people with lower education, often disregarding the basic rules of healthy living, with a prevalence of smoking reaching $60-90 \%$, makes the health situation even worse. The effects of passive smoking are further increased by the small area of apartments, often with less than $10 \mathrm{~m}^{2}$ of space per person. In addition, this population traditionally consumes less fruit and vegetables than the rest of the Czech population. A high frequency of automobile traffic, with the average age of a car reaching almost 15 years, is responsible for extreme pollution from exhaust fumes. The prevalence of respiratory problems and allergies in this region is more than two times higher than in the rest of the Czech Republic.

These facts led us to this study to improve the health of the population at the biggest risk-the children. The first step is to guarantee a short-term stay in an environment with very low pollution (app. $20 \%$ of the original level). It was found that the climatotherapy treatment together with speleotherapy, higher physical activity and improved nutrition resulted in significant medical improvements (Vetvicka et al., 2013a; 2013b; 2013c; Ostojic et al., 2013). As confirmed by other authors (Schuh, 1993), we showed that this improved regime leads to significant improvements of immune functions, lowering of the risk of infections and improvements in the state of current diseases such as respiratory problems or asthma bronchiale (Buka et al., 2006; Davis et al., 2004). In addition, it is clear that a high prevalence of diseases and related deaths resulting in extreme pollution of the environment have high economic effects (Lee et al., 2013; Buka et al., 2006; Linares et al., 2010).

We decided to test the effects of improving the nutritional conditions by supplementing the food with samples with high level of nucleotides. Nucleotides in the form of Nucleic Acids (NA) affect several 
biochemical processes necessary for adequate functions of the living organism. Nucleic acids are monomeric units with both RNA and DNA genetic code (Devresse, 1999; Lerner and Shamir, 2000). They are involved in biosynthesis of glycogen, such as UDPP galactose is involved in synthesis of lactose of UDP glucose. In addition, nucleotides are parts of some coenzymes NAD, FAD and A. In addition, they serve as a biological regulator-cAMP decreases cascade of second messenger, which has a key role in regulation of biological processes and simultaneously represents an extremely important source of energy (Lerner and Shamir, 2000).

An animal model indicated strong effects of 4 weeks supplementation with dietary Nucleotides (dNT) used in drinks on development and quality of terminal ileum (Slizova et al., 2004). In addition, positive effects of dNT on growth and quality of intestinal flora and iron absorption were also demonstrated (Lerner and Shamir, 2000). Human milk contains a higher level of dNT and higher biological availability of iron than bovine milk (Leach et al., 1995; ATSCPSCPFL, 2002; Thorell et al., 1996). In breast-fed infants dNT positively influenced NK cell activity and levels of $\operatorname{IgM}$, IgA and $\operatorname{sg}$ A. The addition of dNT increased the effect of vaccination (Lerner and Shamir, 2000; Leach et al., 1995). dNT also affected liver function and liver regeneration and stimulated production of some components of the complement cascade. On the other hand, dNT deficit (in dNT-free diets) resulted in suppression of some immune reaction, mostly in decrease of IL-2 and IL-3 production and in decrease in numbers of pluripotent stem cells.

It is not surprising, therefore, that this complex role of dNT is currently the focus of extensive interest of both theoretical scientists and clinicians. More and more groups study individual aspects of postnatal development and development of malnourished children living in areas with a contaminated environment. In addition, more and more attention is focused on how to improve immune functions before the start of the expected epidemic of infectious diseases. Supplementation with dNT might offer some help in regeneration processes, normalization of metabolic, physiologic and immune functions in a wide range of patients, from people with high stress to chronically ill patients or the elderly.
In this study, we report our results in supplementation of food with high content of dNT in children from a heavily contaminated environment.

\section{Materials and Methods}

\section{Protocol}

The same protocol that was previously described (Vaclav et al., 2013b) was used throughout this study. Briefly, a randomized trial compared one dose of Imuregen (Uniregen, Nachod, Czech Republic), 2 $\mathrm{mg}$ /day-4 weeks and a placebo in children. A total of 138 children from the sanatorium were enrolled in the 4-week trial testing. The trial was conducted at the Sanatorium EDEL (Zlate Hory, Czech Republic) and the study was approved by the Ethics committees of the Public Health Institute based in Usti nad Labem and Sanatorium EDEL, Zlate Hory, Czech Republic. As a part of the clinical evaluation at the starting point of the study, all children were tested for their height, weight and BMI (Body Mass Index) and its percentile was calculated in relation to age and sex (Table 1). Ninety three children (43 females and 50 males) formed the group supplemented with Imuregen, 45 children (24 females and 21 males) formed the control (placebo) group. This study was performed in agreement with Helsinki declaration (revised version 2000.09.01) and in full compliance with the rules for clinical testing in the Czech Republic. Parental consent was given in all cases. Subjects were routinely evaluated by the medical staff. Imuregen is a food supplement approved by the Chief hygienist of the Czech Ministry of Health.

Tests

In all subjects we obtained saliva at the beginning of the study and at the conclusion of their stay in Sanatorium. We used identical times (between 8 and 9 am) for sampling, so the possible influence of circadian rhythms could be eliminated.

Saliva was collected using a commercial Salivette device (Sarstead, Orsay, France). After two minutes of chewing, the cotton swab was added into a sterile container and centrifuged at $1,000 \mathrm{~g}$ for $15 \mathrm{~min}$ and stored at $-15^{\circ} \mathrm{C}$. We measured the levels of sIgA using nephelometer Siemens BMII as suggested by the manufacturer.

Table 1. Body mass index and its percentile calculated in relation to age and sex

\begin{tabular}{lllllll}
\hline & & $\mathrm{n}$ & Age- mean (SD) & BMI-mean (SD) & Percentiles mean (SD) & BMI <90\% percentiles \\
\hline Nucleotides & Males & 50 & $10,7(3,01)$ & $19,31(3,89)$ & $61,46(31,33)$ & 40 \\
& Females & 43 & $9,55(2,98)$ & $18,03(3,21)$ & $58,86(27,91)$ & 20,9 \\
Placebo & Males & 21 & $11,00(2,18)$ & $18,77(2,99)$ & $58,52(28,09)$ & 23,8 \\
& Females & 24 & $10,62(2,58)$ & $18,89(4,79)$ & $53,33(35,62)$ & 25 \\
\hline
\end{tabular}


All participants absolved at the beginning and at the end of the 6 Min Walk Test (6MWT) based on suggested development, including evaluation of additional parameters such as frequency before and after physical stress, oxygen saturation, interruption of stress due to tiredness. These tests were done $2 \mathrm{~h}$ after the meal. We used recommendations of the American Thoracic Society (ATSCPSCPFL, 2002).

In addition, both weight and height were measured and BMI calculated. BMI levels were subsequently transferred into age percentiles (Body Mass Index for Age Percentiles) according to the CDC (2000). We found that $\mathrm{CDC}$ percentiles are identical to levels found in the population of Czech children of the same age population as determined by The National Institute of Public Health, Prague, Czech Republic. All tested children had minimal physical activity for $2 \mathrm{~h}$ prior to the test with guaranteed no special energetic income. The track used for all testing was long and wide enough to ensure no risk of physical constraints during the test.

At the beginning and the end of the study we also evaluated eNO levels using the American Thoracic Society recommendations (ATSCPSCPFL, 2002) as described in our previous studies (Vetvicka et al., 2013c).

During the whole stay at the Sanatorium EDEL, all children were subjected to the same dietary regime corresponding to their age category.

\section{Statistical Analysis}

Statistical significance was evaluated by a pair t-test and unpaired t-test using a GraphPad Prism 502 software (GraphPad Software, USA).

\section{Results}

Figure 1 summarizes our measurements of the 6MWT in children at the beginning and at the end of their stay in Sanatorium EDEL. We divided all children not only into supplemented and control (placebo) groups, but also based on sex. In the supplemented group, we found a significant increase in 6MWT levels in both the female and male groupsfrom 448 to 470 in males, from 403 to 427 in females. The results in control groups were minor $-12 \mathrm{~m}$ increase in male group, $5 \mathrm{~m}$ increase in female group.

Figure 2 shows the levels of eNO at the beginning and the end of the experiment. The decrease of the level at the end of the stay is significant, particularly in the male group, where we found a decrease from 25.3 to $14.48 \mathrm{ppb}$. In females, the decrease from 15.04 to $11.39 \mathrm{ppb}$ was less pronounced, but still significant. A decrease in the eNO levels was also observed in the control group-nonsignificant in the male group (from 19.08 to $11.57 \mathrm{ppb}$ ) and significant in females (from 14.88 to $11.79 \mathrm{ppb}$ ).

Our findings of $\operatorname{sIgA}$ levels were not significantly changed by diet supplementation with dNT (Fig. 3). In male group, the levels were not changed (app. $185 \mathrm{mg}$ $\mathrm{L}^{-1}$ ). In females, we found a non-significant decrease from $208.9 \pm 34.6$ to $153.8 \pm 36.6 \mathrm{mg} \mathrm{L}^{-1}$. However, in the control group we found a significant decrease in the sIgA levels in saliva of both groups. The decrease in the male group was from $309.7 \pm 33.4\left(\mathrm{mg} \mathrm{L}^{-1}\right)$ to $169.0 \pm 32.9$ and in female group from $217.0 \pm 20.9$ to 102.1 \pm 18.7 . Mean values and SD for all our data are summarized in Table 2.

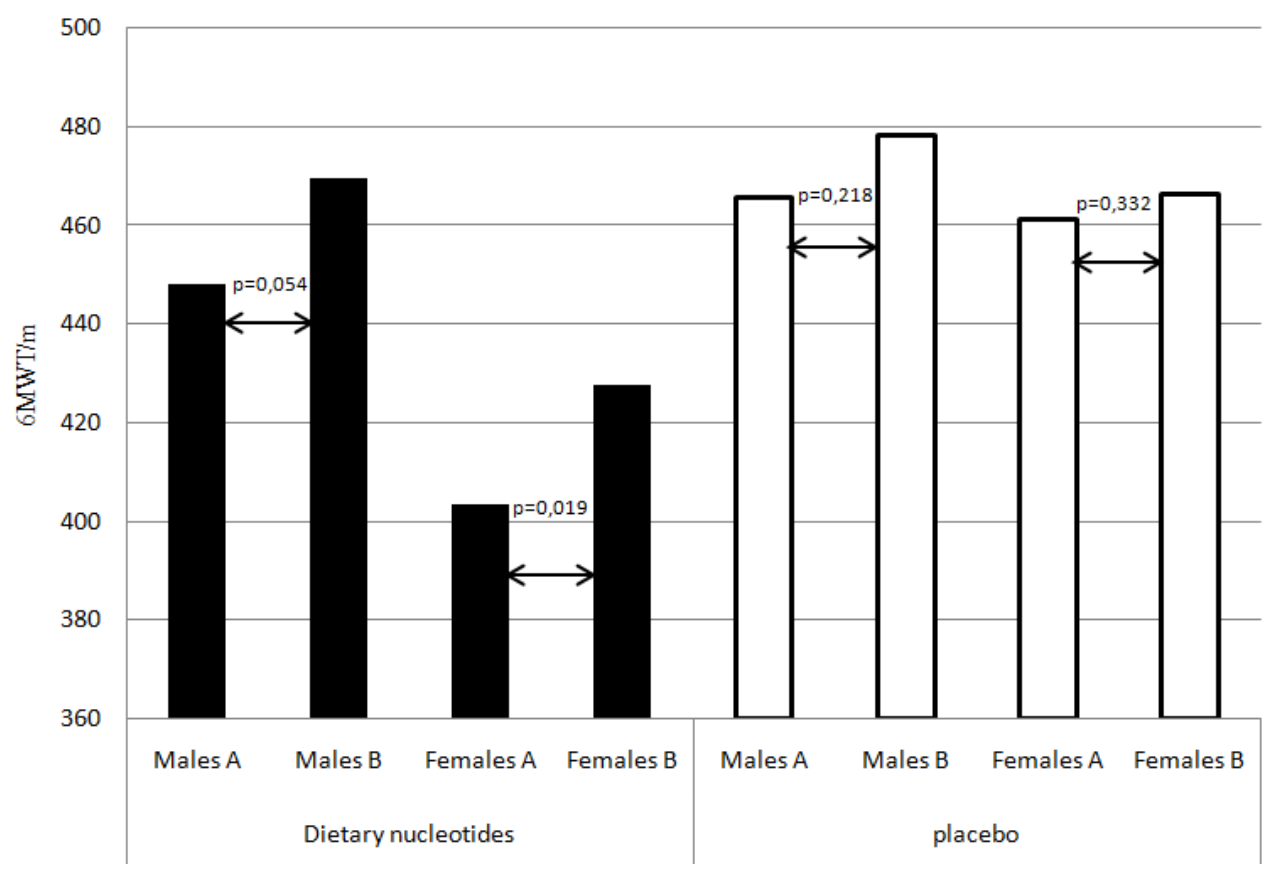

Fig. 1. Effect of supplementation with nucleotides on six minute walk test. A - day 1, B - day 30 


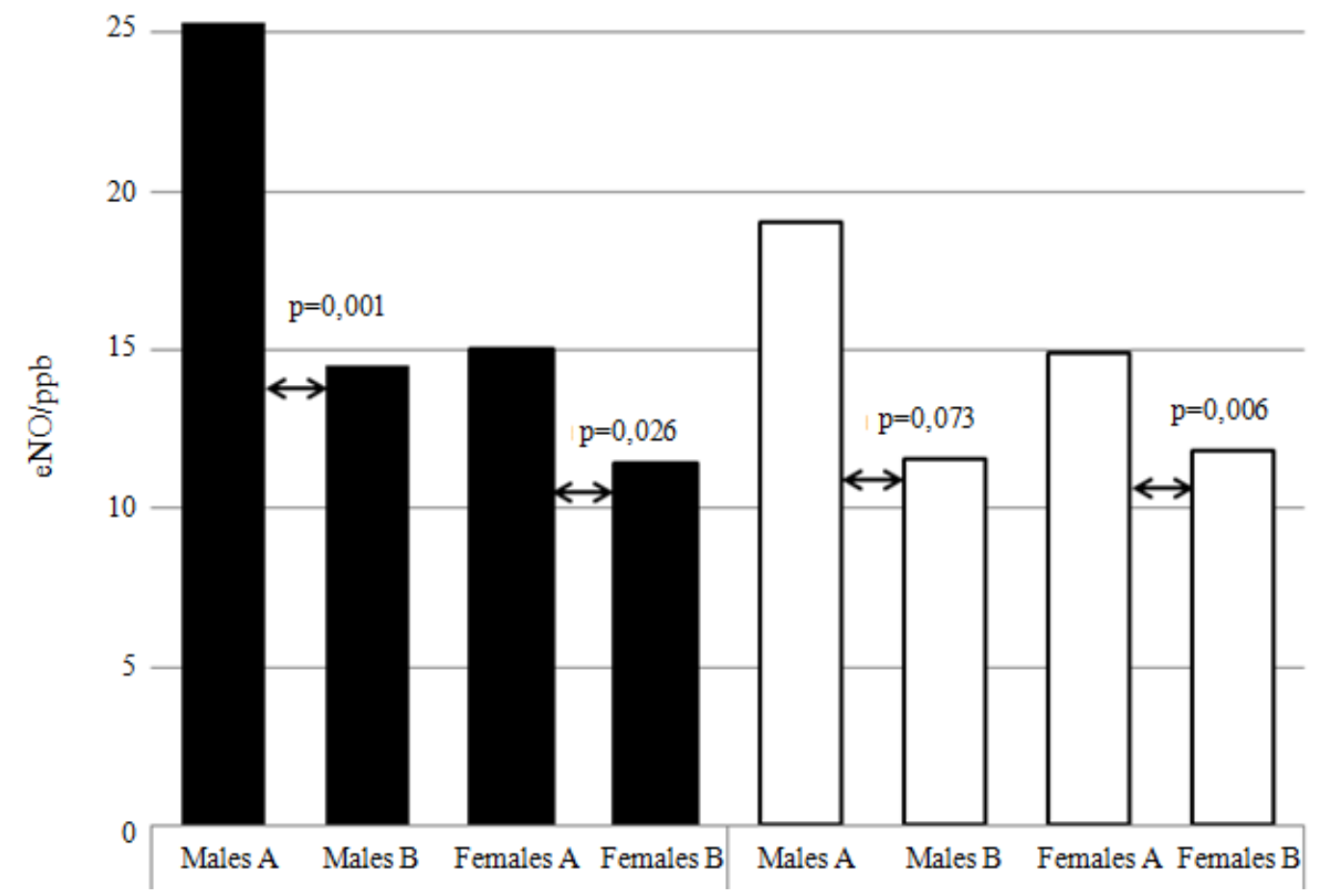

Fig. 2. Levels of eNO in children supplemented with nucleotides or placebo. A-day 1, B-day 30

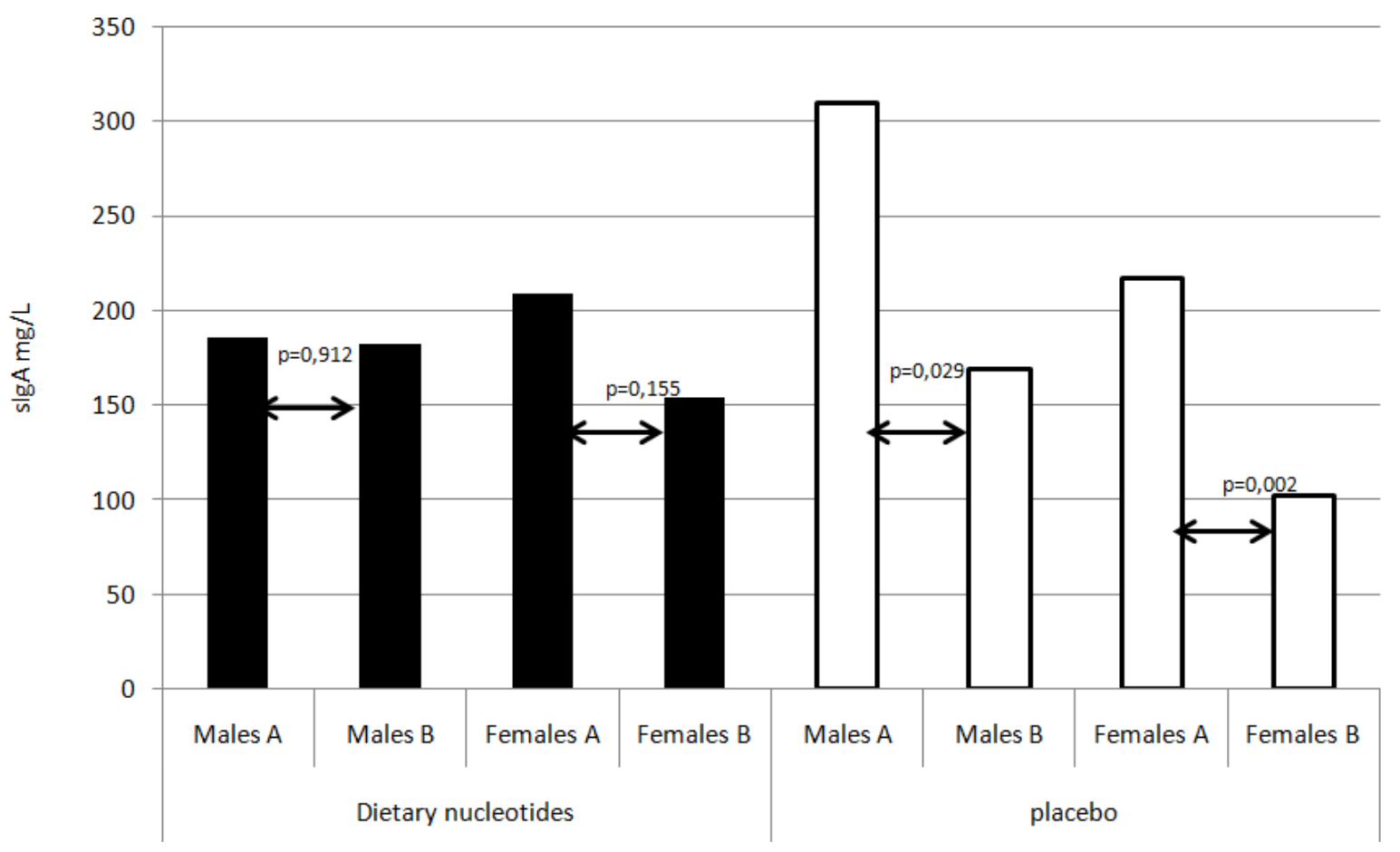

Fig. 3. Effect of supplementation with nucleotides on sIgA levels in saliva. A-day 1, B-day 30 
Table 2. Mean values and SD for all presented data

\begin{tabular}{llllll}
\hline & & $\begin{array}{l}\text { Nucleotides } \\
\text { mean (SD) A }\end{array}$ & $\begin{array}{l}\text { Placebo } \\
\text { mean (SD) B }\end{array}$ & mean (SD) A & mean (SD) B \\
\hline 6 MWT & M & $448(85)$ & $469(85)$ & $465(65)$ & $478(79)$ \\
& F & $403(83)$ & $427(83)$ & $461(95)$ & $466(83)$ \\
SIgA & M & $185,4(22,7)$ & $182,0(32,2)$ & $309,7(33,4)$ & $169,0(32,9)$ \\
& F & $208,9(34,6)$ & $153,8(36,6)$ & $217,3(20,9)$ & $102,1(18,7)$ \\
eNO & M & $25,3(27,2)$ & $14,5(10,6)$ & $19,0(21,1)$ & $11,6(9,7)$ \\
& F & $15,4(17,8)$ & $11,4(8,5)$ & $14,9(13,4)$ & $11,8(10,8)$ \\
\hline
\end{tabular}

\section{Discussion}

Nutritional intervention in the form of food supplementation, with nucleotides, micronutrients, bovine colostrum or glucans, in groups with increased physical challenge have become the main research approach of numerous laboratories (Ostojic et al., 2013; Davis et al., 2004; Gunzer et al., 2012; Ostojic and Obrenovic, 2012; Riera et al., 2013; Stier et al., 2014; Wolvers et al., 2006). It is the results of our quest for an increase of immune reactions not only in athletes, but also in clinical medicine (Ciacio et al., 2014) and in possible renovation of damaged mucosal immunity in children exposed to effects of environmental contaminants (Richter et al., 2014; Vetvicka et al., 2013a; 2013b; 2013c). Based on these trials, several commercial supplements appeared on the market, including Oral Impal from Nestle (Ciacio et al., 2014; Sublingual nucleotides, extract from germinated barley (Ostojic and Obrenovic, 2012) or Immunoactive from Bioiberica Spain (Wolvers et al., 2006).

Our previous studies showed significant effects of $\mathrm{dNT}$ on repairs of mucosa in distal ileus (Slizova et al., 2004). Our data demonstrated that even short term supplementation with dNT affected physical stress related changes by holding the levels of salivary IgA steady, which was strongly suppressed in the control group of children. As high doses of dNT were shown in murine models to increase the allergic reactions (Lerner and Shamir, 2000), it is important to carefully find the optimal dose of dNT. In our current study, we found no dose-related problems, neither clinically or in the laboratory. The effects of dNT supplementation suggests their importance in keeping the optimal quality of physiological functions and supporting some mechanisms of both specific and nonspecific immune reactions. Physical conditions in children supplemented with dNT were significantly improved in the female group. The results of the 6MWT in the male group were at the limit of statistical importance, most probably due to the higher number of obese individuals (over 40\%). A male group showed in relation between BMI percentile and 6MWT statistically significant linear regression at $p<0.0001$ level. We can presume that improvement in $6 \mathrm{MWT}$ levels might be in relation to strong improvements in
eNO values, which were found in all groups. It is clear that the abandoning of the highly polluted conditions (both external and internal such as passive smoking) plays an important role in these findings. Our results confirmed the data achieved by other groups that the 6MWT is a simple, objective and reproducible comparison of the functional capacity both in clinical and rehabilitating programs (ATSCPSCPFL, 2002; Grinnel, 2010; Ulrich et al., 2013).

The salivary samples for evaluations of the level of mucosal immunity were used primary for the easy and non-invasive way to obtain samples. Saliva offers a possibility to test the whole range of important information about the state of mucosal immunity, which can be further used for determination of the general quality of immune reactions. Currently, medical practice offers a wide spectrum of these indicators (Gillum et al., 2013; Koh and Koh, 2007; Krasteva and Kiselova, 2011; Malamud and Rodriguez-Chavez, 2011). We used sIgA because it offers a good indication for testing of the effects of physical stress on mucosal immunity (Gillum et al., 2013; Yi and Moochhala, 2013).

\section{Conclusion}

From our results, we can conclude that supplementation with nucleotides caused significant improvements in all tested reactions.

\section{Acknowledgement}

This study was supported by the Technology Agency of the Czech Republic TACR TA 0202094.

\section{Author's Contributions}

Josef Richter: Participated in all experiments, coordinated the data-analysis and contributed to the writing of teh manuscript.

Vladimir Svozil: Participated in expriments, evaluated all patients.

Vlastimil Kral: Designed the research plan and orgnized the study.

Lucie Rajnohova Dobiasova: Coordinated the patients groups, performed the data analysis. 
Ivana Stiborova: Coordinated the patients groups, performed the data analysis.

Jitka Pohorska: Coordinated the patients groups, performed the data analysis.

Vaclav Vetvicka: Participated in all experiments, wrote and translated the manuscript.

\section{Ethics}

The study was approved by the Ethic committees of the Public Health Institutes of both Sanatorium Edel and ZU Usti nad Labem.

\section{References}

ATSCPSCPFL, 2002. ATS statement: Guidelines for the six-minute walk test. 2002. Am. J. Respir. Crit. Care Med., 166: 111-117. PMID: 12091180

CDC, 2000. Body mass index-for-age percentiles. CDC.

Buka, I., S. Koranteng and A. Osornio-Vargas, 2006. The effects of air pollution on the health of children. Paediatr. Child Health, 11: 513-516.

Ciacio, A., T. Voron, G. Pitau, M. Lewin and R. Adam et al., 2014. Interest of preoperative immunonutrition in liver resection for cancer: Study protocol of the PROPILS trial, a multicenter randomized controlled phase IV trial. BMC Cancer, 14: 1-10. DOI: 10.1186/1471-2407-14-980

Davis, J.M., E.A. Murphy, A.S. Brown, M.D. Carmichael and A. Ghaffar et al., 2004. Effects of moderate exercise and oat $\beta$-glucan on innate immune function and susceptibility to respiratory infection. AJP-Regul. Integr. Comp. Physiol., 286: 366-372. DOI: 10.1152/ajpregu.00304.2003

Devresse, B., 1999. A forgotten but key nutrient for the immune system: Nucleotides. A review and current advances. Guayaquil, Ecuador.

Gillum, T.L., M. Kuennen, C. Gourley, S. Schneider and K. Dokladny et al., 2013. Salivary antimicrobial protein response to prolonged running. Biol. Sport, 3: 3-8. DOI: $10.5604 / 20831862.1029814$

Grinnel, D., 2010. The historical and clinical significance of the 6-minute walk test. Penn StateNoll Laboratory.

Gunzer, W., M. Konra and E. Pail, 2012. Exerciseinduced immunodepression in endurance athletes and nutritional intervention with carbohydrate, protein and fat-what is possible, what is not? Nutrients, 4: 1187-1212. DOI: 10.3390/nu4091187

Koh, D. and G. Koh, 2007. The use of salivary biomarkers in occupational and environmental medicine. Ocup. Environ. Med., 64: 202-210.

DOI: $10.1136 /$ oem.2006.026567
Krasteva, A. and A. Kiselova, 2011. Salivary Acute Phase Proteins as Biomarker in Oral and Systematic Disease. In: Acute Phase Proteins as Early NonSpecific Biomarkers of Human and Veterinary Diseases, Veas, F. (Ed.), InTech pp: 69-88.

Leach, J.L., J.H. Baxter, B.E. Molitor, M.B. Ramstack and M.C. Masor, 1995. Total potentially available nucleosides of human milk by stage of lactation. Am. J. Clin. Nutr., 61: 1224-1230, PMID: 7762521

Lee, S., Y. Chang and S. Cho, 2013. Allergic diseases and air pollution. Asia Pac. Allergy, 3: 145-154. DOI: 10.5415/apallergy.2013.3.3.145

Lerner, A. and R. Shamir, 2000. Nucleotides in infant nutrition: A must or an option. IMAJ, 2: 772-774. PMID: 11344731

Linares, B., J.M. Guizar, N. Amador, A. Garcia and V. Miranda et al., 2010. Impact of air pollution on pulmonary function and respiratory symptoms in children. Longitudinal repeated-measures study. BMC Pulmonary Med., 10: 1-9.

DOI: $10.1186 / 1471-2466-10-62$

Malamud, D. and I. Rodriguez-Chavez, 2011. Saliva as a diagnostic fluid. Dent. Clin. North Am., 55: 159-178. DOI: $10.1016 /$ j.cden.2010.08.004

Ostojic, S.M. and M. Obrenovic, 2012. Sublingual nucleotides and immune response to exercise. J. Int. Soc. Sports Nutrition, 9: 1-4. DOI: $10.1186 / 1550-2783-9-31$

Ostojic, S.M., K. Idrizovic and M.D. Stojanovic, 2013. Sublingual nucleotides prolong run time to exhaustion in young physically active men. Nutrients, 5: 4776-4785. DOI: 10.3390/nu5114776

Richter, J., V. Svozil, V. Král, L. RajnohováDobiášová and I. Stiborová et al., 2014. Clinical trials of yeastderived $\beta$ - $(1,3)$ glucan in children: Effects on innate immunity. Am. Transl. Med., 2: 1-6. DOI: $10.3978 /$ j.issn.2305-5839.2014.02.01

Riera, J., V. Pons, D. Martinez-Puig, C. Chetrit and J.A. Tur et al., 2013. Dietary nucleotide improves markers of immune response to strenuous exercise under a cold environment. J. Int. Soc. Sports Nutrit., 10: 1-8. DOI: 10.1186/1550-2783-10-20.

Schuh, A., 1993. Climatotherapy. Experientia, 49: 947-956. DOI: $10.1007 / \mathrm{BF} 02125641$

Slizova, D., P. Śíma, J. Richter, O. Krs and J. Zavadilová, 2004. Stimulation of ileal epithelium growth and regeneration by dietary nucleotide extracts. Acta Med., 47: 163-166. PMID: 15568732

Stier, H., V. Ebbeskotte and J. Gruenwald, 2014. Immune-modulatory effects of dietary yeast beta1,3/1,6-D-glucan. Nutr. J., 13: 38-38.

DOI: $10.1186 / 1475-2891-13-38$ 
Thorell, L., L. Sjöberg and O. Hernell, 1996. Nucleotides in human milk: Sources and metabolism by the newborn infant. Pediatric Res., 40: 845-852. DOI: 10.1203/00006450-199612000-00012

Ulrich, S., F.F. Hildenbrand, U. Treder, M. Fischler and S. Keusch et al., 2013. Reference values for the 6minute walk test in healthy children and adolescents in Switzerland. BMC Pulm. Med., 13: 49. DOI: $10.1186 / 1471-2466-13-49$

Vetvicka, V., J. Richter, V. Svozil, L. Rajnohova Dobiasova and V. Kral, 2013a. Placebo-driven clinical trials of transfer point glucan \#300 in children with chronic respiratory problems: Antibody production. Am. J. Immunol., 9: 43-47. DOI: 10.3844 /ajisp.2013.43.47

Vetvicka, V., J. Richter, V. Svozil, L. Rajnohová Dobiášová, V. Král, 2013b. Placebo-driven clinical trials of yeast-derived $\beta-(1-3)$ glucan in children with chronic respiratory problems. Ann. Transl. Med., 1: 1-5. DOI: 10.3978/j.issn.2305-5839.2013.07.01
Vetvicka, V., J. Richter, V. Svozil, L. Rajnohova Dobiasova and V. Kral, 2013c. Placebo-driven clinical trials of transfer point glucan \#300 in children with chronic respiratory problems: III. Clinical findings. Am. J. Immunol., 9: 88-93. DOI: 10.3844 /ajisp.2013.88.93

Wolvers, D.A.W., W.M.R. Herpen-Broekman, M.H.G.M. Logman, R.P.J. Van der Wielen and R. Albers, 2006. Effect of a mixture of micronutrients, but not of bovine colostrum concentrate, on immune function parameters in healthy volunteers: A randomized placebo-controlled study. Nutr. J., 5: 28-28. DOI: $10.1186 / 1475-2891-5-28$

Yi, T.C. and S. Moochhala, 2013. Opinion on salivary biomarkers as a measurement for stress and fatigue. Open Biomarkers, 6: 9-14. DOI: $10.2174 / 1875318301306010009$ 